\title{
Feature-Specific Clusters of Neurons and Decision-Related Neuronal Activity
}

\author{
(1)J. Patrick Mayo ${ }^{\star 1}$ and ${ }^{\circledR}$ Bram-Ernst Verhoef ${ }^{\star 1,2}$ \\ ${ }^{1}$ Department of Neurobiology, Harvard Medical School, Boston, Massachussetts 02115, and ${ }^{2}$ Laboratory for Neuro-en Psychophysiology, KU Leuven, \\ Campus Gasthuisberg, BE 3000 Leuven, Belgium \\ Review of Nienborg and Cumming
}

Seminal work in the 1990s by Britten and colleagues provided empirical and computational support for the idea that the activity of single neurons could be used to predict an animal's perceptual decision (Britten et al., 1996). The measure of decision-related activity they introduced, called "choice probability" (CP), is based on signal detection theory and quantifies the discriminability of the distributions of a neuron's firing rates as a function of behavioral choice (e.g., left vs right). CP provides a relatively straightforward way of relating a neuron's firing rate to an animal's decision.

A recent study by Nienborg and Cumming (2014) addresses the question of why some neurons within a brain region exhibit decision-related activity and others do not. Specifically, the authors make the important insight that CPs are more likely to be found when the brain region examined has an anatomical map for the feature tested in the task. These "feature maps" may be cortical columns or clusters of neurons with similar response properties.

Nienborg and Cumming (2006) previously found significant CPs in visual area V2

Received April 13, 2014; revised May 5, 2014; accepted May 7, 2014.

J.P.M. is supported by NIH Grant F32EY022529. B.-E.V. is a postdoctoral research fellow of the Flemish Fund for Scientific Research (FW0). We thank John H.R. Maunsell for helpful comments.

*J.P.M. and B.-E.V. contributed equally to this work.

Correspondence should be addressed to either J. Patrick Mayo or BramErnst Verhoef, Department of Neurobiology, Harvard Medical School, 220 Longwood Avenue, Boston, MA 02115. E-mail: patrick_mayo@hms.harvard.edu or bram-ernst_verhoef@hms.harvard.edu.

DOI:10.1523/JNEUROSCI.1493-14.2014

Copyright $\odot 2014$ the authors $\quad 0270-6474 / 14 / 348385-02 \$ 15.00 / 0$ in monkeys, but not in primary visual cortex (V1), during a depth-discrimination task involving binocular disparity, the property underlying stereopsis. These findings are consistent with the feature-map hypothesis because $\mathrm{V} 2$ has a robust map of disparity, while disparity-sensitive neurons have no strong spatial organization in V1. To test their hypothesis further, Nienborg and Cumming (2014) recorded again in $\mathrm{V} 1$ but now used an orientation discrimination task. If feature maps play a critical role in decision-related activity, one would expect that the columnar organization of orientation-selectivity in V1 yields significant CPs.

Two monkeys were trained to discriminate the orientation of a stimulus by making an eye movement to one of two choice targets, corresponding to preferred and null orientations of the recorded neuron. Rapid sequences of noisy gratings were presented and the authors titrated the amount of noise on each trial to regulate task difficulty. Critically, the orientation signal could be removed completely (random noise only) so that the stimulus was perceptually ambiguous (Nienborg and Cumming, 2014, their Fig. 1). These trials were randomly rewarded, and the monkeys' decisions were presumably driven by small fluctuations in neuronal activity that "pushed" a decision in favor of one orientation over the other.

The main results of Nienborg and Cumming (2014) are consistent with previous investigations of CP (Britten et al., 1996) but, for the first time to our knowledge, demon- strated significant CPs in V1. Overall, when a given neuron fired more during the $0 \%$ signal trials, the monkeys were more likely to choose the preferred orientation target, leading to an average population CP significantly greater than chance (Nienborg and Cumming, 2014, their Fig. 4, left). Alongside their previous disparity study (Nienborg and Cumming, 2006), these results suggest that V1 activity can be used to predict behavior if the neurons are spatially organized for the feature being tested.

Nienborg and Cumming (2014) then performed several analyses to demonstrate that CPs were not trivially related to differences in eye movements, variability in the stimulus sequences, or, to a limited extent, experimental subjects. One concern was that random, small fluctuations in the amount of orientation signal during the nominally $0 \%$ signal trials could yield small differences in CP. Such stimulusdriven effects would undercut the interpretation of CP as purely decision-based changes in neuronal activity. To address this concern, on a subset of recording sessions $(n=21)$, the authors used identical stimulus sequences twice. They found that CPs calculated using the subset of trial pairs for which monkeys responded differently on each presentation were still significantly greater than chance (Nienborg and Cumming, 2014, their Fig. 4, right). Thus, CPs were not trivially explained by stimulus changes.

A final result by Nienborg and Cumming (2014) further strengthens their hypothesis. One of the two monkeys in this 
study of orientation had previously been examined in their work on disparity discrimination (Nienborg and Cumming, 2006) (it is unclear which monkey is in both studies and whether single-electrodes or linear-electrode arrays were used across studies). A direct comparison of V1 activity in the same monkey but different tasks revealed that $\mathrm{CPs}$ in $\mathrm{V} 1$ were significantly greater in the orientation task, arguing against the possibility that $\mathrm{CP}$ differences reflect differences in subjects across tasks.

In their Discussion, Nienborg and Cumming (2014) suggest that certain properties associated with neuronal clusters provide the foundation for the previously proposed relationship between decision-related activity and correlated trial-by-trial fluctuations in the neuronal responses. These "noise correlations" may contribute to decisionrelated activity because correlated noise does not average out and therefore can drive decisions based on a weighted sum of activity within a population. Yet significant noise correlations are not sufficient to explain decision-related activity. For example, when the correlated activity within a population of neurons with similar stimulus preferences is also shared with a population of neurons with opposite preferences, a decision based on the difference in average activity between the populations would eliminate the correlated activity through subtraction, thereby preventing it from influencing the decision. Hence, to explain decision-related activity, some activity should be shared within a population but not across populations (Shadlen et al., 1996).

However, this conclusion leaves many unresolved experimental questions. Specifically, it is unclear why neuronal clusters should share more activity within than between them. One possibility is that different clusters have different shared inputs, as suggested by the stronger noise correlations between nearby neurons and neurons with similar feature preferences (Lee et al., 1998). Alternatively, theoretical work has shown that clustered connections in recurrent neuronal networks can induce state transitions in activity that are correlated within but anticorrelated between clusters (LitwinKumar and Doiron, 2012). Furthermore, feedback signals such as those related to feature attention have often been suggested as a source of differential noise correlations and CP (Nienborg and Cumming, 2009). However, one study (Cohen and Maunsell, 2011) empirically measured the impact of feature attention on noise correlations and found that feature attention actually decreases noise correlations, mainly between neurons with similar tuning properties (i.e., putative clusters), thereby changing the noisecorrelation structure in a way that seems to oppose instead of facilitate CP. Thus, what properties of neuronal clusters underlie the noise correlation pattern and $\mathrm{CP}$ is an important question for future research.

Nevertheless, the findings of Nienborg and Cumming $(2006,2014)$ are consistent with a relation between decision-related activity and cortical clustering. A remaining question is whether neurons in parts of the cortex with heterogeneous feature preferences have lower CPs, as the authors' hypothesis suggests. To answer this question, both CPs and feature maps need to be measured. A more stringent test would entail measuring decision-related activity within the same cortical area for neurons in populations with either homogeneous or heterogeneous feature preferences. Such experiments seem feasible in parts of extrastriate cortex where clustered neurons for, say, faces or shapes are often flanked by neurons with similar feature selectivities but mixed preferences.

Interestingly, studies using electrical microstimulation to determine the influence of neuronal activity on perception often stimulate neurons in the same clusters as those in which significant $\mathrm{CPs}$ have been observed (Salzman et al., 1990; DeAngelis et al., 1998). Microstimulation in featurespecific clusters influences the monkeys' perceptual choices and appears to increase the perceptual evidence in favor of the preferred stimulus of the stimulated neurons. These findings suggest that neurons with decision-related activity contribute to rather than merely correlate with task performance.

Although Nienborg and Cumming (2014) did not illustrate changes in CP over time, significant decision-related activity often arises soon after stimulus onset when neurons become selective for the task's stimulus feature (Nienborg and Cumming, 2006). After its peak, the CP often stays relatively high. Notably, studies using reaction-time tasks or psychophysicalreverse-correlation analyses (Nienborg and Cumming, 2009) have shown that monkeys mostly rely on the perceptual evidence presented at the start of the trial, a point in time where significant $\mathrm{CPs}$ are already present. The early component of the decision-related activity may therefore primarily reflect a feedforward influence on task performance (or, perhaps, a pre-established behavioral bias), consistent with the microstimulation results discussed above. The later, sustained part of the $\mathrm{CP}$ likely happens when the monkey has already made its decision so it may encompass working-memory and feedback activity. Consequently, the "feedforward versus feedback" debate in the $\mathrm{CP}$ literature might not have a straightforward solution. Instead, these observations suggest that the source of decision-related activity could lie in both feedforward and feedback activity, where the weight of each component varies as a function of poststimulus time.

The hypothesized relationship between decision-related activity and cortical clustering links several research topics in system neuroscience, including decision making, noise correlations, microstimulation, and cortical architecture. The authors' hypothesis therefore provides a fertile ground for further research. The merits of forthcoming research could be high, as they may provide us with insights into the origin of decision-related activity and, more broadly, into the functional interplay between feedforward and feedback neuronal circuitry.

\section{References}

Britten KH, Newsome WT, Shadlen MN, Celebrini S, Movshon JA (1996) A relationship between behavioral choice and the visual responses of neurons in macaque MT. Vis Neurosci 13:87-100. CrossRef Medline

Cohen MR, Maunsell JH (2011) Using neuronal populations to study the mechanisms underlying spatial and feature attention. Neuron 70 : 1192-1204. CrossRef Medline

DeAngelis GC, Cumming BG, Newsome WT (1998) Cortical area MT and the perception of stereoscopic depth. Nature 394:677-680. CrossRef Medline

Lee D, Port NL, Kruse W, Georgopoulos AP (1998) Variability and correlated noise in the discharge of neurons in motor and parietal areas of the primate cortex. J Neurosci 18 : 1161-1170. Medline

Litwin-Kumar A, Doiron B (2012) Slow dynamics and high variability in balanced cortical networks with clustered connections. Nat Neurosci 15:1498-1505. CrossRef Medline

Nienborg H, Cumming BG (2006) Macaque V2 neurons, but not V1 neurons, show choicerelated activity. J Neurosci 26:9567-9578. CrossRef Medline

Nienborg H, Cumming BG (2009) Decisionrelated activity in sensory neurons reflects more than a neuron's causal effect. Nature 459:89-92. CrossRef Medline

Nienborg H, Cumming BG (2014) Decisionrelated activity in sensory neurons may depend on the columnar architecture of cerebral cortex. J Neurosci 34:3579-3585. CrossRef Medline

Salzman CD, Britten KH, Newsome WT (1990) Cortical microstimulation influences perceptual judgements of motion direction. Nature 346:174-177. CrossRef Medline

Shadlen MN, Britten KH, Newsome WT, Movshon JA (1996) A computational analysis of the relationship between neuronal and behavioral responses to visual motion. J Neurosci 16:1486-1510. Medline 